\title{
Cortical network dynamics of perceptual decision-making in the human brain
}

\author{
Markus Siegel ${ }^{1,2 *}$, Andreas K. Engel $^{3}$ and Tobias H. Donner ${ }^{4}$ \\ 1 Centre for Integrative Neuroscience, University of Tübingen, Tübingen, Germany \\ 2 The Picower Institute for Learning and Memory, Massachusetts Institute of Technology, Cambridge, MA, USA \\ ${ }^{3}$ Department of Neurophysiology and Pathophysiology, University Medical Center Hamburg-Eppendorf, Hamburg, Germany \\ ${ }^{4}$ Department of Psychology, University of Amsterdam, Amsterdam, Netherlands
}

\section{Edited by:}

Thilo Womelsdorf, Robarts Research Institute, Canada

\section{Reviewed by:}

Jean-Philippe Lachaux, INSERM,

France

Thilo Womelsdorf, Robarts Research

Institute, Canada

\section{${ }^{*}$ Correspondence:}

Markus Siegel, Centre for Integrative Neuroscience, University of Tübingen, 72076 Tübingen, Germany.

e-mail:markus.siegel@uni-tuebingen.de; Tobias H. Donner, Department of Psychology, University of Amsterdam, 1018 WB Amsterdam, Netherlands. e-mail:t.h.donner@uva.nl
Goal-directed behavior requires the flexible transformation of sensory evidence about our environment into motor actions. Studies of perceptual decision-making have shown that this transformation is distributed across several widely separated brain regions. Yet, little is known about how decision-making emerges from the dynamic interactions among these regions. Here, we review a series of studies, in which we characterized the cortical network interactions underlying a perceptual decision process in the human brain. We used magnetoencephalography to measure the large-scale cortical population dynamics underlying each of the sub-processes involved in this decision: the encoding of sensory evidence and action plan, the mapping between the two, and the attentional selection of task-relevant evidence. We found that these sub-processes are mediated by neuronal oscillations within specific frequency ranges. Localized gamma-band oscillations in sensory and motor cortices reflect the encoding of the sensory evidence and motor plan. Large-scale oscillations across widespread cortical networks mediate the integrative processes connecting these local networks: Gamma- and beta-band oscillations across frontal, parietal, and sensory cortices serve the selection of relevant sensory evidence and its flexible mapping onto action plans. In sum, our results suggest that perceptual decisions are mediated by oscillatory interactions within overlapping local and large-scale cortical networks.

Keywords: perceptual decision-making, neuronal oscillations, neuronal synchronization, magnetoencephalography, sensorimotor integration, attention, gamma-band, beta-band
We often need to select our actions based on perceptual interpretations of noisy, incomplete, or ambiguous sensory information about our environment. Imagine driving on the highway on a rainy night. To discern if the cars in front of you slowed down, you try to judge whether their brake lights are looming. After accumulating the sparse sensory information for a few moments you eventually decide to hit the brake.

During sensory-guided behaviors such as this one, perceptual states are flexibly mapped onto our motor actions, a process referred to as perceptual decision-making (Figure 1; Gold and Shadlen, 2001, 2007; Usher and McClelland, 2001; Heekeren et al., 2008; Tosoni et al., 2008). Dynamic interactions between multiple processing stages of the brain's sensorimotor pathways lie at the heart of such decision processes. Invasive recordings in monkeys performing simple sensorimotor tasks have characterized the detailed functional properties of individual neurons in these processing stages (Schall, 2001; Glimcher, 2003; Romo and Salinas, 2003; Gold and Shadlen, 2007; Kable and Glimcher, 2009). These studies have shown that neurons in sensory, parietal, and frontal cortices, are involved in encoding the sensory evidence, accumulating this evidence over time, and planning the ensuing motor action. These results have inspired circuit models of the large-scale interactions between these processing stages (Wang, 2002, 2008; Mazurek et al., 2003; Lo and Wang, 2006; Gold and Shadlen, 2007). However, until recently, these large-scale interactions have only rarely been characterized directly (Heekeren et al., 2004; Pesaran et al., 2008), but mainly been inferred (Wang, 2002, 2008; Mazurek et al., 2003; Lo and Wang, 2006; Gold and Shadlen, 2007).

A hallmark of the neuronal interactions underlying perceptual decisions is their flexibility. The mapping of sensory evidence onto motor actions is not a stereotyped reflex but depends strongly on the decision-maker's current behavioral goals. In different contexts, the exact same sensory input can be mapped onto different actions. Similarly, the same action can be selected in the face of different sensory inputs. To account for this flexibility, theories of cognitive control postulate "top-down" signals that selectively bias the flow of information in the brain's sensorimotor pathways, such that only the sensory and motor neuronal populations relevant for the goal at hand communicate with one another, whereas goal-irrelevant pathways are shut down (Miller and Cohen, 2001). These top-down signals are thought to originate from prefrontal and posterior parietal association cortices (Miller and Cohen, 2001; Corbetta and Shulman, 2002).

Most current models of perceptual decision-making conceptualize the decision formation as a feedforward integration process from sensation to action (Usher and McClelland, 2001; Mazurek et al., 2003; Smith and Ratcliff, 2004). However, the actual cortical circuits implementing this process likely engage in highly recurrent interactions (Lamme and Roelfsema, 2000; Wang, 2008). Such recurrent interactions are mediated by the abundant bi-directional 


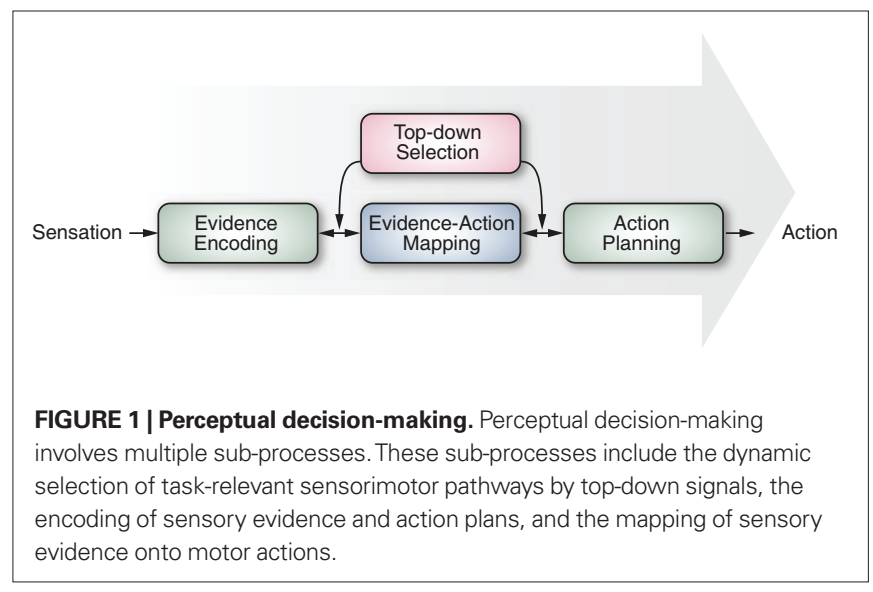

cortico-cortical connections (Felleman and Van Essen, 1991). For example, sensorimotor association regions of the parietal and frontal cortex commonly display highly similar response profiles during decision formation (Kim and Shadlen, 1999; Shadlen and Newsome, 2001; Pesaran et al., 2008). Furthermore, neuronal activity even at the earliest sensory processing stages (such as visual cortical areas V1 and V2) does not merely reflect the sensory evidence but also the result of the perceptual decision process, likely via feedback from downstream areas (Donner et al., 2008a,b; Nienborg and Cumming, 2009). In sum, even simple perceptual decisions emerge from recurrent and flexible interactions between widely distributed regions of the cerebral cortex. How can we get a glimpse on these interactions?

\section{NEURONAL OSCILLATIONS: A WINDOW ON THE DYNAMICS OF DECISION-MAKING}

Magnetoencephalography (MEG) combined with source reconstruction techniques is ideally suited to simultaneously monitor the dynamics of neuronal population activity across widely separated regions of the human cerebral cortex and to characterize the dynamic interactions between these regions. Moreover, the exquisite temporal resolution of MEG allows for characterizing the fine temporal structure of cortical population activity. A pervasive feature of this activity is that it displays oscillations across a broad range of frequencies and spatial scales (Buzsaki and Draguhn, 2004).

A growing body of evidence suggests that such neuronal oscillations play an important functional role in cortical information processing. Coherent oscillations, within and between cortical regions may flexibly regulate the interactions among distributed neuronal populations (Engel et al., 2001; Salinas and Sejnowski, 2001; Varela et al., 2001; Fries, 2005; Haider and McCormick, 2009). On the one hand, synchronization of presynaptic spikes within a local cortical region enhances their functional impact on postsynaptic neurons in a super-additive fashion (Alonso et al., 1996; König et al., 1996; Azouz and Gray, 2003). On the other hand, the phase-alignment of presynaptic spikes to postsynaptic oscillations modulates their efficiency in driving postsynaptic spikes (Fries, 2005; Womelsdorf et al., 2007; Haider and McCormick, 2009). Both types of synchrony may serve as flexible mechanisms to control the gain of local and long-range neuronal communication.
Furthermore, neuronal oscillations at different frequencies may provide valuable mechanistic information about the interactions between groups of neurons (Siegel and Donner, 2010). First, they may index interactions within specific neural circuits. Local cortical oscillations in the gamma-band $(30-80 \mathrm{~Hz})$ provide an intriguing example for a spectral signature for which the detailed circuit-level mechanisms are becoming increasingly clear. Second, more generally, neuronal oscillations may reflect different types of neural interactions. In particular, they may reflect reverberating activity within local as well as large-scale cortical networks (Wang, 2003). Such reverberation may mediate persistent cortical activity and recurrent cortical processing (Siegel et al., 2000, 2009; Tallon-Baudry et al., 2001; Pesaran et al., 2002; Van Der Werf et al., 2008), which, in turn, may underlie the protracted accumulation of sensory evidence during decision-making. Third, neuromodulators such as norepinephrine or acetylcholine profoundly shape the synaptic interactions between cortical neurons. Thus, the spectral signature of neuronal oscillations may also reflect the effects of neuromodulators (Steriade, 2000; Rodriguez et al., 2004), which, in turn, play important roles in decision-making (Aston-Jones and Cohen, 2005; Yu and Dayan, 2005). In sum, the spectral signature of cortical population activity may provide rich information about the detailed neuronal circuit dynamics underlying decision-making. Such information might only be weakly expressed in the spiking activity of individual neurons.

\section{DISSECTING A PERCEPTUAL DECISION PROCESS}

How does the flexible mapping of sensory "evidence" onto action plans emerge from these neuronal circuit dynamics? We addressed this question in a series of MEG experiments in humans, dissecting a single perceptual decision into its component sub-processes. In all experiments subjects judged visual motion signals contained in dynamic random-dot patterns and reported their perceptual interpretation of these stimuli by button-press. By adjusting the fraction of coherently moving dots in the random-dot patterns, we could precisely and specifically control the strength of the sensory feature of interest, i.e., visual motion (Figure 2A; Scase et al., 1996). Moreover, this enabled us to titrate the sensory evidence to the subjects' individual detection threshold. Near psychophysical threshold, perceptual choices vary form trial to trial in the face of identical sensory inputs (Green and Swets, 1966). We could exploit these intrinsic fluctuations to isolate the neuronal mechanisms underlying the varying choices without confounding variations of the sensory input. This task also allowed us to focus our analyses on well-characterized cortical regions, which had been identified in previous single-unit recordings in monkeys (Newsome et al., 1989; Britten et al., 1993; Kim and Shadlen, 1999; Shadlen and Newsome, 2001; Roitman and Shadlen, 2002; Gold and Shadlen, 2007) and fMRI studies in humans (Rees et al., 2000; Shulman et al., 2001; Serences and Boynton, 2007). We used an adaptive spatial filtering technique (linear beamforming; Van Veen et al., 1997; Gross et al., 2001) to estimate the dynamics of neuronal population activity within and among these cortical processing stages.

\section{GAMMA-BAND ACTIVITY IN VISUAL CORTEX REFLECTS SENSORY EVIDENCE}

We set out by investigating the input stage of the decision process, i.e., the encoding of sensory evidence for coherent motion (Siegel et al., 2007). Subjects were presented with dynamic random-dot 


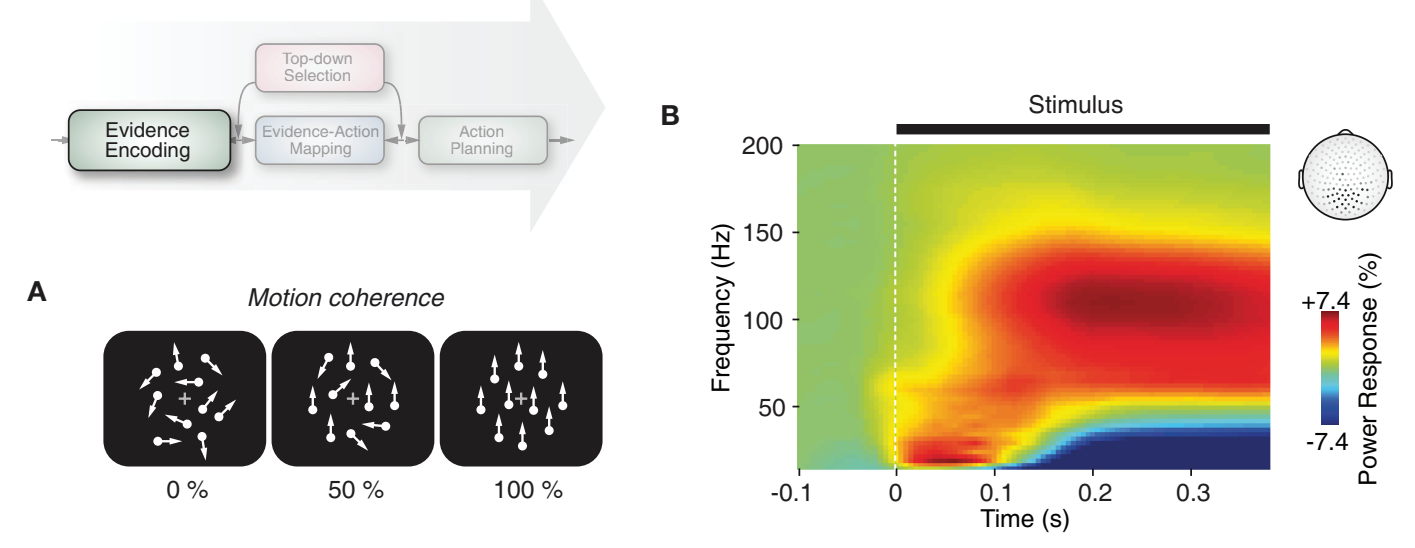

C

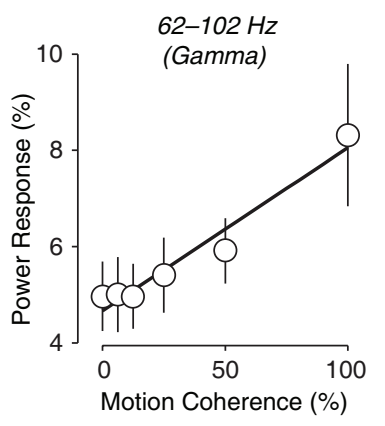

FIGURE 2 | Gamma-band activity in visual cortex reflects the encoding of sensory evidence. (A) Schematic illustration of dynamic random-dot patterns at three levels of motion coherence. The percentage of coherently moving dots controls the strength of the motion signal and thus the strength of sensory evidence for the motion-discrimination task. (B) Time-frequency representation of neural responses to dynamic random-dot patterns recorded at MEG sensors overlying visual cortex. Responses were averaged across six logarithmically scaled levels of motion coherence ranging from 0 to $100 \%$ (see panel C) and across seven subjects. (C) Gamma-band activity $(62-102 \mathrm{~Hz})$ over visual cortex

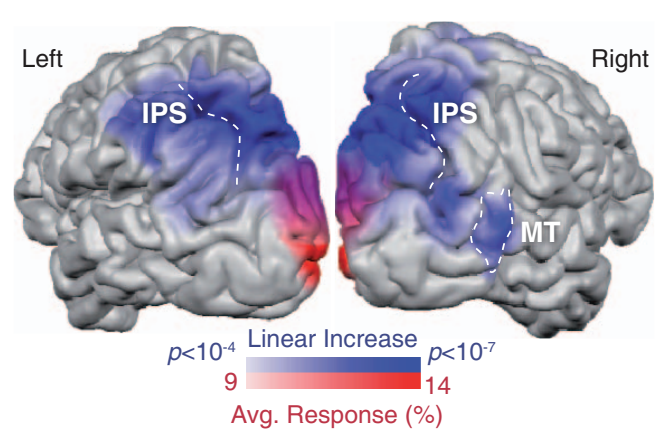

during stimulus viewing (100-500 ms post stimulus onset) increases approximately linearly with motion coherence. The solid line indicates the significant linear effect ( $p<0.01 ; n=7$ subjects). (D) Cortical distribution of the average gamma-band response $(62-102 \mathrm{~Hz})$ across all levels of motion coherence (red shading) and of the linear increase of gamma-band activity with motion coherence (blue shading). Data is shown for one representative subject. See Siegel et al. (2007) for full details and data from all individual subjects (IPS, intraparietal sulcus; MT, middle temporal area). Reprinted and modified with permission from Siegel et al. (2007). patterns at various different levels of motion coherence (Figure 2A) and, for each stimulus, reported the net motion direction (up vs. down) by button-press.

The moving stimuli induced robust population responses with a characteristic dynamic signature at sensors overlying visual cortex (Figure 2B). Following a transient low-frequency response (reflecting the visual evoked field), the MEG power increased in a broad high-frequency range (about 40-150 Hz), accompanied by a decrease in the low-frequency range below $40 \mathrm{~Hz}$. Both of these response components were sustained as long as the stimuli were on the screen. The response in a narrower gamma-band from about 60 to $100 \mathrm{~Hz}$ increased approximately linearly with the strength of visual motion (Figure 2C). This frequency-specific parametric effect on the stimulus response was remarkably consistent across all individuals. We also observed a weaker and less consistent opposite relationship in the lower frequency range (alpha- and betabands). Here, activity monotonically decreased with increasing motion strength.

The modulation of the gamma-band activity with motion strength was specifically expressed in those regions of extrastriate visual cortex that process visual motion. Across all levels of motion coherence, early visual areas around the calcarine displayed the strongest average gamma-band response (Figure 2D, red shading). By contrast, the robust increase in gamma-band activity with motion strength predominated in motion-sensitive extrastriate cortical regions, such as area MT and other regions in occipitoparietal cortex (Figure 2D, blue shading). These results suggest that local gamma-band activity in functionally specialized regions of the visual cortex reflects the local processing of specific features of the visual environment. This is consistent with evidence from other studies in animals (Frien et al., 2000; Siegel and König, 2003; Kayser and König, 2004; Henrie and Shapley, 2005; Liu and Newsome, 2006; Berens et al., 2008) and humans (Hall et al., 2005; Hoogenboom et al., 2005; Vidal et al., 2006; Wyart and Tallon-Baudry, 2008) demonstrating visual gamma-band activity as well as its dependence on stimulus strength and various stimulus features. This is further consistent with the finding that pre-stimulus fluctuations of gamma-band activity in visual cortex have an impact on visual detection decisions (Wyart and TallonBaudry, 2009). Thus, local gamma-band activity in sensory cortex is a proxy for the encoding of sensory evidence during perceptual decision-making. 


\section{BUILD-UP OF CORTICAL POPULATION ACTIVITY IN MOTOR CORTEX REFLECTS THE EVOLVING ACTION PLAN}

The spectral signature of sensory encoding was highly consistent with the signature of action encoding at the cortical output stage of the decision process. In another experiment, the dynamic randomdot patterns shown on each trial contained either pure dynamic noise, or a weak motion signal titrated to each subject's individual detection threshold (Donner et al., 2009). Subjects formed a decision about the presence or absence of this target motion signal. Stimuli were presented for a fixed duration of $2 \mathrm{~s}$ and followed by a blank delay, after which subjects reported their choice (Figure 3A). In this task, psychophysical coherence detection thresholds decrease over stimulus durations of more than $3 \mathrm{~s}$ in humans. Thus, presenting motion signals at the subjects' individual detection threshold ensured that each subject accumulated the evidence for motion until the end of the stimulus interval.

To track the subjects' evolving plan to report either "target present" or "target absent" within the brain, we mapped these two perceptual choices onto button-presses with different hands. This allowed us to exploit the contralateral bias of the cortical motor system (i.e., stronger activity in the hemisphere contralateral to the motor movement). Just before the execution of the motor response, gamma-band activity (about $60-100 \mathrm{~Hz}$ ) in primary motor (M1) and premotor (PMd) cortices was selectively enhanced contralateral to the button-press (Figures 3A,B). As for the stimulus-driven responses in visual cortex discussed above, this gamma-band enhancement was accompanied by a decrease of low-frequency activity (about $10-35 \mathrm{~Hz}$ ) with two distinct peaks in the alpha- and beta-bands. This antagonistic modulation of gamma-band and low-frequency activity in motor and premotor cortex is in line with previous intracranial and MEG studies (Crone et al., 1998a,b; Pfurtscheller et al., 2003; Cheyne et al., 2008).

Crucially, both components of the effector-selective activity were not only expressed after the completion of the decision process (i.e., after stimulus offset), but they built up gradually during stimulus viewing, while subjects were forming their decision (Figure 3C). Both components reliably predicted subjects' final choice on single trials seconds before its execution. Thus, local gamma-band activity and an accompanying suppression of low-frequency activity in motor cortex reflect the evolving action selection in this protracted decision process.

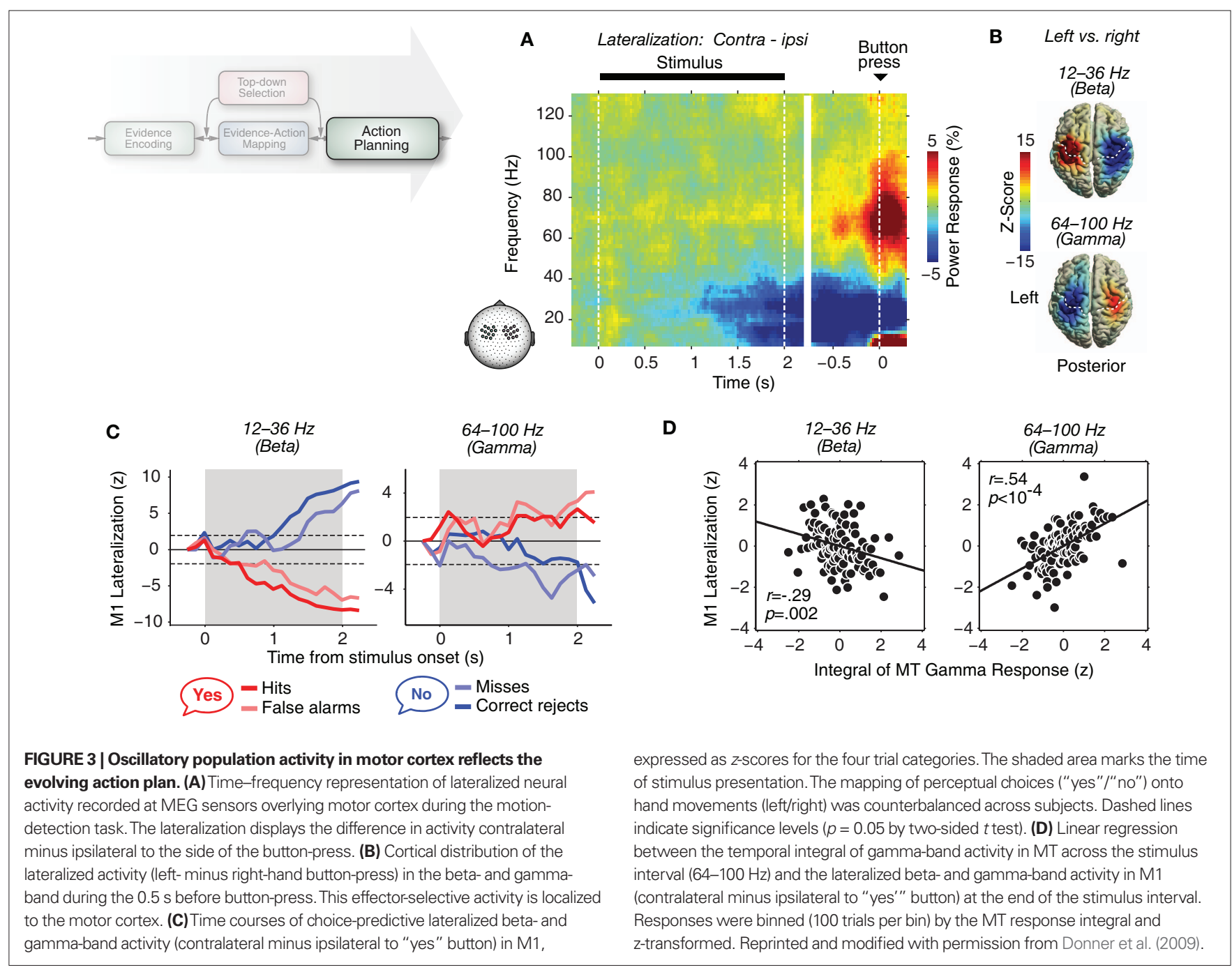


We interpret the gamma-band enhancement as a more direct marker of the local neuronal representation than the suppression of low-frequency activity, for a number of reasons. First, for both, sensory and motor responses, gamma-band activity is positively correlated with local processing while low-frequency activity shows the opposite relationship. Second, intracranial recordings from motor cortex show that gamma-band modulations are typically anatomically more focused than the low-frequency suppressions (Crone et al., 1998a,b; Pfurtscheller et al., 2003). This also explains why these premotor gamma-band modulations are more difficult to detect in extracranial signals like EEG and MEG than in direct invasive recordings from the cortical surface. Third, gamma-band activity in motor cortex typically shows a stronger effector-selectivity (Crone et al., 1998a,b; Pfurtscheller et al., 2003; Rickert et al., 2005; Cheyne et al., 2008). Fourth, in our own data, the premotor gamma-band modulations were more closely linked to the ongoing decision process, as discussed in the next section.

\section{CHOICE-PREDICTIVE ACTIVITY IS DRIVEN BY INTEGRATED SENSORY EVIDENCE}

The results discussed so far established frequency-specific neuronal signatures for the task-relevant sensory evidence (visual motion: gamma-band activity in MT) and the evolving motor plan (button-press: gamma-band activity in motor cortex). A large body of evidence suggests that the evidence encoded in sensory cortex is integrated across time during the decision process (Kim and Shadlen, 1999; Shadlen and Newsome, 2001; Usher and McClelland, 2001; Roitman and Shadlen, 2002; Smith and Ratcliff, 2004; Gold and Shadlen, 2007). The simultaneous monitoring of sensory evidence and motor plan with MEG provides an ideal opportunity to test this model. If the choice-predictive build-up of activity in motor cortex is driven by the temporal integral of evidence for motion delivered by MT, then the trial-to-trial fluctuations of this motor activity should be predicted by the trial-to-trial fluctuations of the integrated MT activity.

This is what we found (Figure 3D; Donner et al., 2009). The temporal integral of gamma-band activity in MT predicted the choice-predictive motor activity in the gamma- and beta-bands. The opposite sign of correlation reflected the opposite sign of movement-selective activity in these bands (Figures 3A-C). This correlation was stronger for premotor activity in the gamma-band, suggesting that the local gamma-band activity is more tightly linked to the local neuronal representation than low-frequency activity (see above). Notably, the correlation between choice-predictive motor activity and gamma-band activity in MT was much weaker if not the integral of MT activity but only its instantaneous activity was taken into account. Further, the correlation between MT-integral and motor activity did not merely emerge at the end of the decision process (the stimulus offset), but was continuously expressed during stimulus viewing, irrespective of whether the decision-process resulted in a "target present" or a 'absent" choice. These observations establish a first direct link between the neuronal dynamics of sensory evidence and motor plan during perceptual decision-making and provide strong evidence for the temporal accumulation model.

Importantly, our results do not imply that the temporal integration process takes place in the motor cortex. Any processing stage between MT and the motor cortex may be involved in accumulating the sensory evidence delivered by MT. Posterior parietal and prefrontal association cortex have long been known to exhibit persistent neuronal activity (Miller and Cohen, 2001; Wang, 2001; Pessoa et al., 2002; Machens et al., 2005). More recently, these regions have also been implicated in the temporal integration of sensory evidence (Wang, 2002; Heekeren et al., 2004, 2008; Gold and Shadlen, 2007; Kaiser et al., 2007).

The results discussed in this section show that the outcome of the temporal integration process is continuously routed through all the way even to primary motor cortex. This conclusion is consistent with a previous microstimulation study in the monkey's oculomotor system (Gold and Shadlen, 2000) and suggests a highly continuous flow of information in the sensorimotor pathways (Kim and Shadlen, 1999; Shadlen and Newsome, 2001; Horwitz et al., 2004; Gold and Shadlen, 2007). This contradicts the traditional notion of a "central executive" that first completes the decision process before sending its end result to the output stage.

\section{FRONTAL-PARIETAL BETA-BAND OSCILLATIONS PREDICT THE ACCURACY OF EVOLVING DECISION}

How can one obtain a more direct handle on the neuronal mechanism of the evidence-action mapping per se? A powerful strategy is to exploit the fact that, at low levels of sensory evidence, subjects often make incorrect choices. A substantial fraction of these performance fluctuations is thought to be driven by variability at the decision stage (Shadlen et al., 1996). Specifically, we reasoned that the more efficiently the evidence-action mapping mechanism operates on any given trial, the more likely subjects will make a correct choice on that trial. Thus, we compared neuronal activity in the motion-detection task between correct and incorrect trials (Donner et al., 2007). Enhanced activity in a narrow "low beta" frequency range (about 12-24 Hz) predicted the correctness of the subjects' upcoming choice (Figure 4A). This effect specifically emerged during the stimulus interval, that is, while subjects were accumulating the sensory evidence and while activity was building up in their motor cortex. This performance-predictive beta-band activity was expressed in a widespread network across the cerebral cortex, predominantly in posterior parietal and dorsolateral prefrontal cortex and, less strongly, in visual (e.g., area MT) and motor cortex (Figure 4B).

Beta-band activity in these regions was higher before "hits" than "misses" on target present trials and higher before "correct rejects" than "false alarms" on target absent trials (Figure 4C). Thus, this beta-band activity predicted the accuracy (correct vs. error) but not the content (target present vs. target absent) of the upcoming choice. This suggests that the widespread, performance-predictive beta-band oscillations reflect the computations governing the decision process rather than neuronal representations involved at its different stages (deCharms and Zador, 2000). In other words, the trial-to-trial fluctuations of beta-band activity do not reflect fluctuations of the neuronal representations of sensory evidence, decision variables, or action plans, but rather fluctuations of the flexible mapping between these representations.

What is the specific functional role of the performance-predictive beta-band oscillations? Several recent studies reported analogous beta-band oscillations across frontal and parietal cortices during visuomotor tasks in humans (Gross et al., 2004; Hipp et al., 2011) 


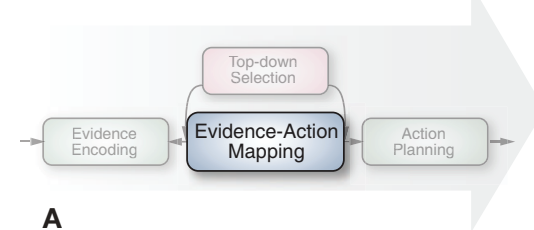

A

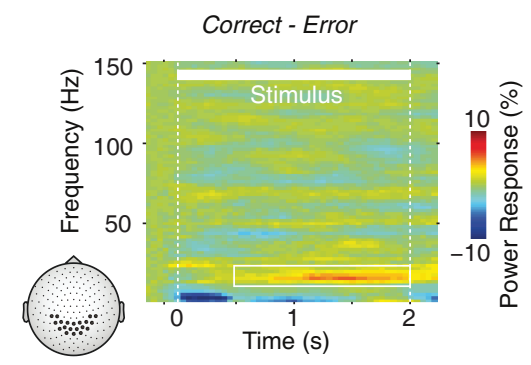

B

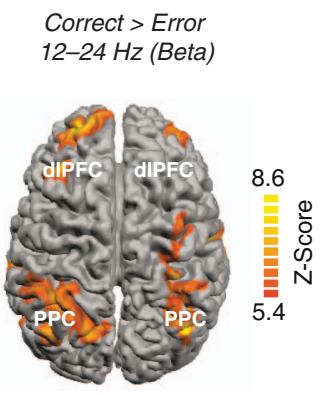

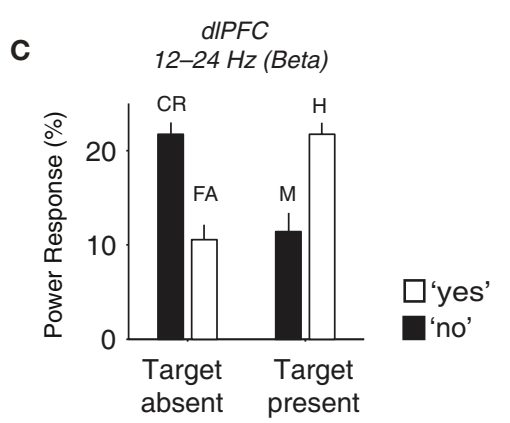

FIGURE 4 | Frontoparietal beta-band activity predicts decision accuracy. (A) Time-frequency representation of the difference in neural activity between correct and error trials recorded at MEG sensors overlying parietal cortex during the motion-detection task. (B) Cortical distribution of the performance-predictive beta-band activity (correct $>$ error; $p<0.001$ corrected; dIPFC, dorsolateral prefrontal cortex; PPC, posterior parietal cortex). (C) Mean beta-band responses in dIPFC, for each stimulus-choice combination. Data is shown for one representative subject. See Donner et al. (2007) for full details and data from all individual subjects (CR, correct rejects; FA, false alarms; $\mathrm{M}$, misses; $\mathrm{H}$, hits). Reprinted and modified with permission from Donner et al. (2007). and monkeys (Pesaran et al., 2002, 2008; Buschman and Miller, 2007, 2009). The performance-predictive frontoparietal beta-band activity in our task likely does not reflect trial-to-trial fluctuations of the subjects' level of arousal or top-down selective attention. Fluctuations of arousal are typically slow, spanning several trials. Thus, such fluctuations should be expressed during the pre-stimulus baseline interval. Also fluctuations of selective attention are often evident during the pre-stimulus baseline (Ress et al., 2000). By contrast, there was no evidence for performance-predictive betaband activity before stimulus onset, but this activity only occurred during the decision period of the trial. Furthermore, several studies discussed in the next section have demonstrated very different spectral profiles for top-down selective attention in similar cortical regions.

Nevertheless, the performance-predictive beta-band activity bears functional similarity with attention. It reflects a mechanism that, similar to attention, governs the accuracy of a flexible sensorimotor transformation. The beta-band oscillations may reflect reverberant activity within and among visual, frontoparietal, and motor cortices (Wang, 2002; Engel and Fries, 2010). This largescale network reverberation may help maintain and accumulate sensory evidence during the decision formation (Donner et al., 2007). Such activity may also reflect the maintenance of the sensorimotor mapping rule between accumulated sensory evidence and action. Further experiments are required to assess these not mutually exclusive scenarios.

\section{TOP-DOWN ATTENTION IS MEDIATED BY LOCAL AND LARGE-SCALE SYNCHRONIZATION DURING DECISION FORMATION}

Perceptual decision-making is not a passive response, but an active and flexible process. The evidence-action mapping can be flexibly adapted to our current behavioral goal. For example, this mapping is often selective for only those sources of sensory evidence that are relevant for achieving our goal. Think again about driving your car at night. You may not only decide to hit the brake when traffic slows down but also when you want to exit the highway. In the former situation you pay attention to the cars in front of you. By contrast, in the latter case your motor response is based on other parts of the available visual information such as the road signs signaling your exit.

The top-down selection of sensory evidence is commonly referred to as "selective attention." Oscillatory synchronization of neuronal activity has frequently been proposed as a candidate mechanism of attention (Gruber et al., 1999; Worden et al., 2000; Engel et al., 2001; Fries et al., 2001; Taylor et al., 2005; Vidal et al., 2006; Saalmann et al., 2007; Wyart and Tallon-Baudry, 2008). In another MEG experiment (Siegel et al., 2008), we thus investigated how selective attention modulated cortical population dynamics along the different stages of the sensorimotor pathway. On each trial, subjects were visually cued to covertly shift their focus of attention to either the left or right visual hemifield, where two random-dot patterns were simultaneously presented after a delay (Figure 5A). Subjects then judged the net-direction of motion of the cued stimulus while ignoring the uncued stimulus. Again, we adjusted the levels of motion coherence to the subjects' individual psychophysical thresholds. This ensured that the task was attentionally demanding. To isolate the neuronal correlates of spatially selective attention, we contrasted modulations of neuronal activity between the hemispheres contralateral and ipsilateral to the attended hemifield.

Visual-spatial attention modulated local oscillatory population activity throughout the visuomotor pathway in a spatially selective fashion (Figure 5B). We observed attentional modulations of local synchrony in visual cortical areas consistent with previous studies (Fries et al., 2001; Bichot et al., 


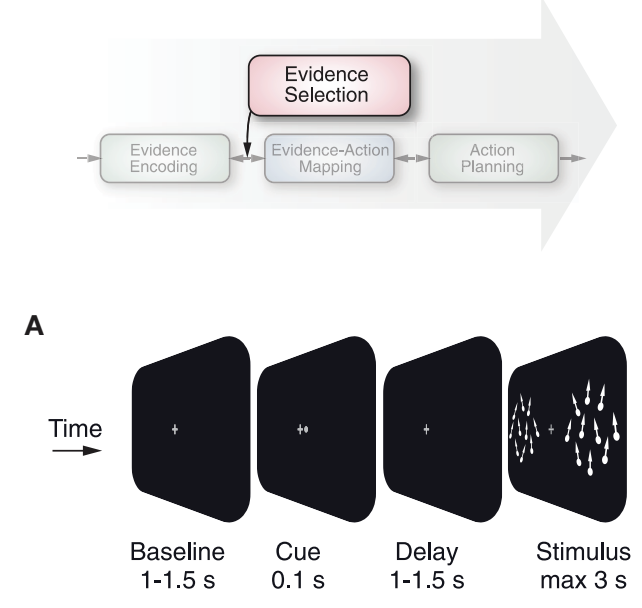

B

C

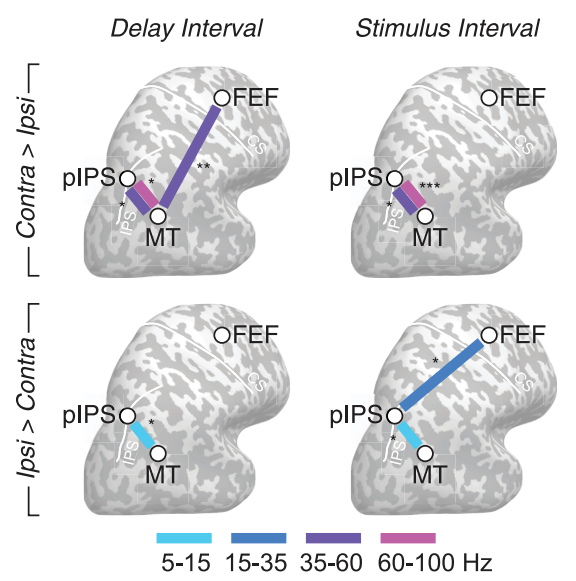

FIGURE 5 | Local and large-scale synchrony reflects the attentional selection of sensory evidence. (A) Schematic illustration of the stimulus display during the spatially cued motion-discrimination task. (B) Attentional modulation of local oscillatory population activity in four regions of interest (marked on the inflated hemispheres to the left). Attentional modulations are displayed as predictive indices that quantify the probability with which an ideal observer can predict the direction of attention (left vs. right) from lateralization of neural responses between the left and right hemispheres on a single trial level. Predictive indices larger or smaller than chance level (0.5) correspond to an attentional enhancement or suppression of activity in the hemisphere contralateral to the attended hemifield, respectively. (C) Attentional modulation
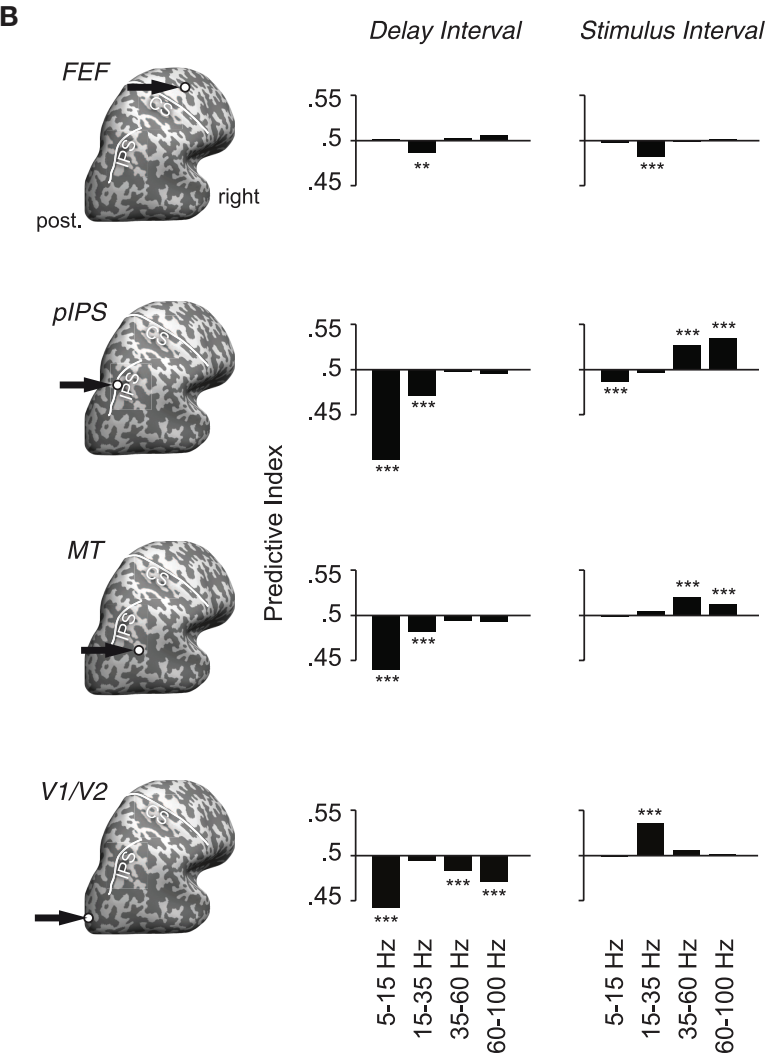

of frequency-specific long-range synchronization. Panels in the upper row display between which cortical regions spatial attention significantly enhanced synchronization in the hemisphere contralateral as compared to ipsilateral to the attended visual hemifield. Panels in the lower row depict corresponding attentional reductions of synchronization. Colors indicate the frequency bands of significantly modulated long-range synchrony. All attentional modulations are displayed separately for the delay interval (750-0 ms before stimulus onset) and stimulus interval (100-500 ms after stimulus onset). $\left({ }^{*} p<0.05,{ }^{*} p<0.01\right.$,

$*^{* *} p<0.001$ corrected; FEF, frontal eye field; PIPS, posterior intraparietal sulcus; MT, middle temporal area; $V 1 N 2$, pericalcarine cortex). Reprinted and modified with permission from Siegel et al. (2008).

lations differed markedly between temporal intervals with more prominent low-frequency suppressions before stimulus onset and enhanced high-frequency activity during stimulation. Thus, the large-scale investigation of attentional modulations throughout the visuomotor pathway revealed a remarkable regional and temporal specificity of these modulations.

Having established that attention modulates oscillatory population activity within visual cortex and attentional control regions, we investigated if the attentional selection of the behaviorally relevant stimulus may be mediated by long-range synchronization between these processing stages. Membrane-potential oscillations establish periodic windows of enhanced excitability (Haider and McCormick, 2009).Attention may thus involve the phase-alignment of presynaptic spikes to postsynaptic oscillations to dynamically 
regulate the information flow between areas (Engel et al., 2001; Fries, 2005). Consistent with this hypothesis, we found that attention modulated long-range oscillatory synchronization between frontoparietal control regions (IPS and FEF) and visual cortex (MT; Figure 5C). In contrast to the effects on local population activity, attentional modulations of long-range synchrony were comparatively stimulus independent (compare Figures 5C,B). Before and during stimulus presentation, attention selectively enhanced longrange synchronization in the gamma-band (35-100 Hz) and suppressed low-frequency synchronization in the alpha- and beta-band (5-35 Hz) in the hemisphere processing the attended stimulus. Thus, visuospatial attention established a spatially selective pattern of long-range synchronization between frontoparietal and early sensory processing stages.

This modulation of synchrony may allow specific neuronal populations within control regions to communicate top-down signals to specific populations in visual cortex without the control regions being necessarily involved in the sensorimotor transformation per se. Alternatively, phase coherence throughout the sensorimotor pathway may enhance the feedforward routing of selected information to the motor stage. Recent results on synchrony between FEF and V4 in monkeys (Gregoriou et al., 2009) suggest that both mechanisms are involved. During sustained attention, synchrony primarily mediates feedforward interactions, while feedback interactions are dominating during shifts of attention. In summary, our results suggest that long-range oscillatory synchronization between frontoparietal control regions and early sensory processing stages mediates the selective routing of sensory information required for the perceptual decision at hand.

\section{LOCAL AND LARGE-SCALE OSCILLATORY NETWORK INTERACTIONS MEDIATE PERCEPTUAL DECISION-MAKING}

The experiments described above provide a first comprehensive characterization of the cortical dynamics underlying a perceptual decision. We delineated the spectral signatures and cortical distributions of its component sub-processes. This revealed multiple local and large-scale oscillatory networks mediating the decision (Figure 6).

At the peripheral stages of the decision process (visual and motor cortex), local gamma-band activity consistently reflected the encoding of sensory evidence and the evolving motor plan (Figure 6A). Both, sensory and motor-related gamma-band activity was accompanied by a suppression of local low-frequency activity. Converging evidence suggest that local cortical gamma-band oscillations are generated by locally recurrent interactions between excitatory neurons and GABAergic interneurons (Siegel et al., 2000; Hasenstaub et al., 2005; Bartos et al., 2007; Gieselmann and Thiele, 2008; Cardin et al., 2009). These excitatory-inhibitory feedback loops become engaged during enhanced processing within local cortical networks and play an important role in shaping the structure of local neuronal representations (Heeger et al., 1996; Shapley et al., 2003). Thus, gamma-band activity within visual and motor cortex indexes the local circuit interactions underlying the encoding of sensory evidence and motor plans. Furthermore, local excitatory-inhibitory interactions, and thus local gamma-band activity, may not only be driven by feedforward inputs to the local network (e.g., visual input in visual cortex) but also by feedback signals originating from higher cortical regions (e.g., attentional signals in visual cortex; Fries et al., 2001; Siegel et al., 2008).

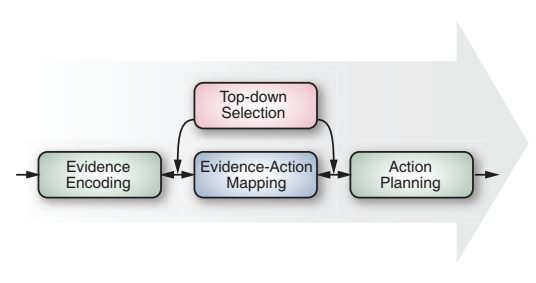

A

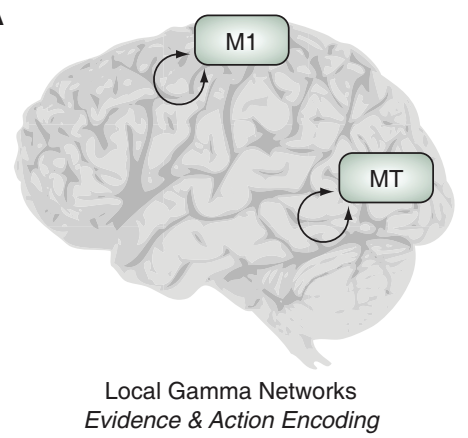

B

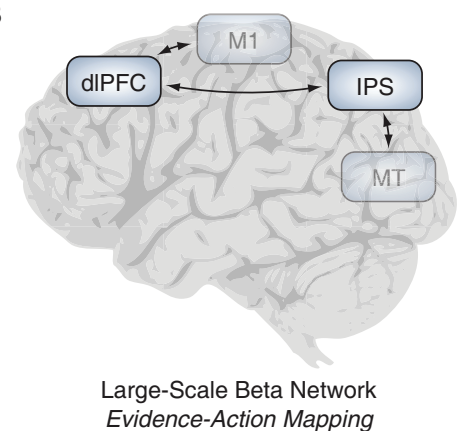

C

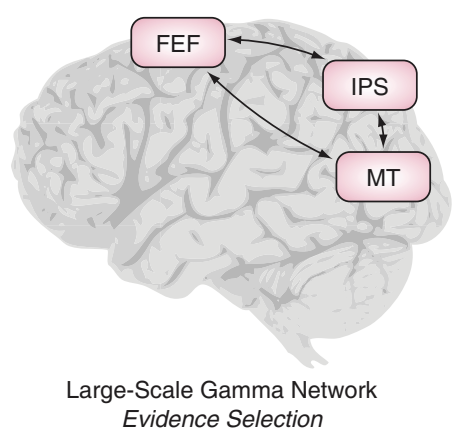

FIGURE 6 | Oscillatory interactions in local and large-scale cortical networks mediate perceptual decision-making. (A) Local gamma-band activity in visual (MT) and motor cortex (M1) reflects the encoding of sensory evidence and motor plan, respectively. Top-down processes modulate this local activity. (B) Large-scale beta-band activity in a network centered on prefrontal (dIPFC) and parietal cortex
(IPS) is involved in mapping the sensory evidence onto motor plans. (C) Large-scale gamma-band synchrony in a network across prefrontal (FEF), parietal (FEF), and visual cortex (MT) mediates the attentional selection of task-relevant sensory evidence (M1, primary motor cortex; MT, middle temporal area; dIPFC, dorsolateral prefrontal cortex; IPS, intraparietal sulcus; FEF, frontal eye field). 
The whole-brain coverage of MEG allowed us to also identify oscillatory interactions in two large-scale cortical networks as central components of the decision process. First, beta-band activity in a widespread network centered on prefrontal and parietal association cortices seems to be instrumental in linking sensory evidence to motor plans (Figure 6B). Specifically, this large-scale beta-band activity may mediate the active maintenance of past evidence during its accumulation and/or its flexible routing to motor plans. Second, top-down attentional selection of particular sources of sensory evidence was associated with enhanced gammaband synchronization in another large-scale network, spanning frontoparietal attentional control regions and extrastriate visual areas (Figure 6C). Gamma-band synchronization within this network may mediate the attentional selection of the behaviorally relevant visual inputs.

Remarkably, the large-scale beta- and gamma-band networks partially overlapped in the posterior parietal cortex. This suggests that overlapping or even identical groups of neurons within this region may simultaneously participate in these two distinct largescale networks. The posterior parietal cortex may thus serve as a hub integrating the large-scale processes mediated by these networks. Thus, shifting the perspective from the responses of individual cortical neurons onto their embedding into large-scale functional networks may help understand the functional role of posterior parietal cortex for both, stimulus and action selection (Gold and Shadlen, 2001; Andersen and Buneo, 2002; Sugrue et al., 2005).

The distinct spectral signatures of the demonstrated local and large-scale oscillatory interactions likely reflect the different mechanisms underlying these oscillations. As outlined above, the detailed mechanisms underlying local gamma-band activity are becoming increasingly clear. By contrast, comparatively little is known about the mechanisms shaping the spectral signatures of oscillatory interactions within large-scale cortical networks. These signatures likely reflect global network properties such as the number of involved regions, synaptic distances, and conduction delays as well as local cellular properties such as the time-constants of various membrane conductances (Kopell et al., 2000). Furthermore, the spectral signatures of large-scale interactions may also depend on local oscillatory networks embedded within these larger networks. Locally generated oscillations such as local gamma-band activity may provide the basic temporal scaffolding necessary to synchronize activity across larger networks. Large-scale spectral signatures may thus reflect global network properties, cellular properties, as well as the spectral signatures of embedded local oscillations. In sum, the spectral signatures of local and large-scale neuronal oscillations provide valuable information about the specific circuit-level interactions underlying decision-making.

\section{CONCLUSIONS AND FUTURE DIRECTIONS}

Taken together, our results begin to unravel how neuronal interactions within overlapping cortical networks form flexible interfaces between perception and action during simple, well-controlled cognitive tasks. On the one hand, fast oscillations within local networks subserve the representation of sensory evidence and motor plans at the periphery of the decision process. On the other hand, coherent oscillations across large-scale cortical networks mediate the integrative processes connecting these local representations, such as the selection of relevant sensory evidence and its flexible mapping onto action plans.

We are still far from understanding the mechanisms that underlie these oscillatory interactions during decision formation. We propose three directions of future research that may be crucial for unraveling these mechanisms. A first fundamental question is if the large-scale oscillatory interactions between distant brain regions are regulated by a superordinate "control region," or if they primarily emerge from distributed network interactions. Evidence suggests that the prefrontal cortex may flexibly boost or block such interregional couplings, as necessary for the task at hand (Miller and Cohen, 2001). Indeed, our results revealed the FEF, a prefrontal region, as an integral part of the large-scale network controlling the flexible evidence selection. However, these interregional couplings may also emerge in a decentralized, self-organizing fashion. Future studies should distinguish between these alternatives.

Second, it remains to be determined how neuronal processing is integrated among distinct large-scale oscillatory networks. The partially overlapping beta- and gamma-band networks discussed above provide an intriguing example. Cross-frequency interactions, such as phase-amplitude and phase-phase coupling between different rhythms, may play an important role for linking different oscillatory processes. Indeed, a growing body of evidence demonstrates different types of cross-frequency interactions in cortical population activity (Schack et al., 2002; Lakatos et al., 2005; Palva et al., 2005; Canolty et al., 2006; Jensen and Colgin, 2007; Siegel et al., 2009). Future studies should investigate if these cross-frequency couplings mediate interactions between different oscillatory networks and if these interactions between networks are modulated in a task-dependent fashion.

Third, the cortical systems involved in decision-making are known to be under profound influence of ascending neuromodulatory systems (Montague et al., 2004; Aston-Jones and Cohen, 2005; Yu and Dayan, 2005). These neuromodulatory systems are thus in an ideal position to dynamically shape these large-scale cortical interactions. Indeed evidence suggests that neuromodulatory systems play an important role in orchestrating oscillatory cortical activity (Rodriguez et al., 2004; Goard and Dan, 2009). Studying the impact of these neuromodulators on the patterns of large-scale cortical activity during decision-making will open up a new dimension in this field of research.

The results described here show that frequency-specific cortical population dynamics, as measured with MEG, provide a powerful window into the distributed dynamics of decision-making in the working human brain. Future studies should exploit this window to further our understanding of what it means "to decide."

\section{ACKNOWLEDGMENTS}

We thank Mike X. Cohen, Simon van Gaal, Floris de Lange, and Jefferson Roy for comments. This work was supported by grants from the European Union (NEST-PATH-043457, HEALTH-F2-2008-200728), the German Research Foundation (GRK 1247/1/2, SFB TRR58), the German Federal Ministry of Education and Research (01GW0561, Neuroimage Nord), and the German Academy of Sciences Leopoldina (THD: BMBF-LPD 9901/8-136). 


\section{REFERENCES}

Alonso, J. M., Usrey, W. M., and Reid, R. C. (1996). Precisely correlated firing in cells of the lateral geniculate nucleus. Nature 383, 815-819.

Andersen, R. A., and Buneo, C. A. (2002). Intentional maps in posterior parietal cortex. Annu. Rev. Neurosci. 25, 189-220.

Aston-Jones, G., and Cohen, J. D. (2005). An integrative theory of locus coeruleus-norepinephrine function: adaptive gain and optimal performance. Annu. Rev. Neurosci. 28, 403-450.

Azouz, R., and Gray, C. M. (2003). Adaptive coincidence detection and dynamic gain control in visual cortical neurons in vivo. Neuron 37, 513-523.

Bartos, M., Vida, I., and Jonas, P. (2007). Synaptic mechanisms of synchronized gamma oscillations in inhibitory interneuron networks. Nat. Rev. Neurosci. 8, 45-56.

Berens, P., Keliris, G. A., Ecker, A. S., Logothetis, N. K., and Tolias, A. S. (2008). Feature selectivity of the gamma-band of the local field potential in primate primary visual cortex. Front. Neurosci. 2: 37. doi: 10.3389/ neuro.01.037.2008

Bichot, N. P., Rossi, A. F., and Desimone, R. (2005). Parallel and serial neural mechanisms for visual search in macaque area V4. Science 308, 529-534.

Britten, K. H., Shadlen, M. N., Newsome, W. T., and Movshon, J. A. (1993). Responses of neurons in macaque MT to stochastic motion signals. Vis. Neurosci. 10, 1157-1169.

Buschman, T. J., and Miller, E. K. (2007). Top-down versus bottom-up control of attention in the prefrontal and posterior parietal cortices. Science 315, 1860-1862.

Buschman, T. J., and Miller, E. K. (2009). Serial, covert shifts of attention during visual search are reflected by the frontal eye fields and correlated with population oscillations. Neuron 63 , 386-396.

Buzsaki, G., and Draguhn, A. (2004). Neuronal oscillations in cortical networks. Science 304, 1926-1929.

Canolty, R. T., Edwards, E., Dalal, S. S., Soltani, M., Nagarajan, S. S., Kirsch, H. E., Berger, M. S., Barbaro, N. M., and Knight, R. T. (2006). High gamma power is phase-locked to theta oscillations in human neocortex. Science 313, 1626-1628.

Cardin, J. A., Carlen, M., Meletis, K., Knoblich, U., Zhang, F., Deisseroth, K., Tsai, L. H., and Moore, C. I. (2009). Driving fast-spiking cells induces gamma rhythm and controls sensory responses. Nature. 459, 663-667.

Cheyne, D., Bells, S., Ferrari, P., Gaetz, W., and Bostan, A. C. (2008). Self-paced movements induce high-frequency gamma oscillations in primary motor cortex. Neuroimage 42, 332-342.

Corbetta, M., and Shulman, G. L. (2002). Control of goal-directed and stimulusdriven attention in the brain. Nat. Rev . Neurosci. 3, 201-215.

Crone, N. E., Miglioretti, D. L., Gordon, B., and Lesser, R. P. (1998a). Functional mapping of human sensorimotor cortex with electrocorticographic spectral analysis. II. Event-related synchronization in the gamma band. Brain $121(\mathrm{Pt}$ 12), 2301-2315.

Crone, N. E., Miglioretti, D. L., Gordon, B., Sieracki, J. M., Wilson, M. T., Uematsu, S., and Lesser, R. P. (1998b). Functional mapping of human sensorimotor cortex with electrocorticographic spectral analysis. I. Alpha and beta event-related desynchronization. Brain $121(\mathrm{Pt}$ 12), 2271-2299.

deCharms, R. C., and Zador, A. (2000). Neural representation and the cortical code. Annu. Rev. Neurosci. 23, 613-647.

Donner, T. H., Sagi, D., Bonneh, Y. S., and Heeger, D.J. (2008a). Global response in human V1 time-locked to perceptual report of a local stimulus change. Soc. Neurosci. Abstr. 871.818.

Donner, T. H., Sagi, D., Bonneh, Y. S., and Heeger, D. J. (2008b). Opposite neural signatures of motion-induced blindness in human dorsal and ventral visual cortex. J. Neurosci. 28, 10298-10310.

Donner, T. H., Siegel, M., Fries, P., and Engel, A. K. (2009). Buildup of choicepredictive activity in human motor cortex during perceptual decision making. Curr. Biol. 19, 1581-1585.

Donner, T. H., Siegel, M., Oostenveld, R., Fries, P., Bauer, M., and Engel, A. K. (2007). Population activity in the human dorsal pathway predicts the accuracy of visual motion detection. J. Neurophysiol. 98, 345-359.

Engel, A. K., and Fries, P. (2010). Betaband oscillations-signalling the status quo? Curr. Opin. Neurobiol. 20, 156-165.

Engel, A. K., Fries, P., and Singer, W. (2001). Dynamic predictions: oscillations and synchrony in top-down processing. Nat. Rev. Neurosci. 2, 704-716.

Felleman, D. J., and Van Essen, D. C. (1991). Distributed hierarchical processing in the primate cerebral cortex. Cereb. Cortex 1, 1-47.

Frien,A., Eckhorn, R., Bauer, R., Woelbern, T., and Gabriel, A. (2000). Fast oscillations display sharper orientation tuning than slower components of the same recordings in striate cortex of the awake monkey. Eur. J. Neurosci. 12, 1453-1465.
Fries, P. (2005). A mechanism for cognitive dynamics: neuronal communication through neuronal coherence. Trends Cogn. Sci. 9, 474-480.

Fries, P., Reynolds, J. H., Rorie, A. E., and Desimone, R. (2001). Modulation of oscillatory neuronal synchronization by selective visual attention. Science 291, 1560-1563.

Gieselmann, M.A., and Thiele, A. (2008) Comparison of spatial integration and surround suppression characteristics in spiking activity and the local field potential in macaque V1. Eur. J. Neurosci. 28, 447-459.

Glimcher, P. W. (2003). The neurobiology of visual-saccadic decision making. Annu. Rev. Neurosci. 26, 133-179.

Goard, M., and Dan, Y. (2009). Basal forebrain activation enhances cortical coding of natural scenes. Nat Neurosci. 12 1444-1449.

Gold, J. I., and Shadlen, M. N. (2000). Representation of a perceptual decision in developing oculomotor commands. Nature 404, 390-394.

Gold, J. I., and Shadlen, M. N. (2001). Neural computations that underlie decisions about sensory stimuli. Trends Cogn. Sci. 5, 10-16.

Gold, J. I., and Shadlen, M. N. (2007). The neural basis of decision making. Annu. Rev. Neurosci. 30, 535-574.

Green, D. M., and Swets, J.A. (1966). Signal Detection Theory and Psychophysics. New York: Wiley.

Gregoriou, G. G., Gotts, S. J., Zhou, H., and Desimone, R. (2009).High-frequency, long-range coupling between prefrontal and visual cortex during attention. Science 324, 1207-1210.

Gross, J., Kujala, J., Hamalainen, M. Timmermann, L., Schnitzler, A., and Salmelin, R. (2001). Dynamic imaging of coherent sources: studying neural interactions in the human brain. Proc Natl. Acad. Sci. U.S.A. 98, 694-699.

Gross, J., Schmitz, F., Schnitzler, I., Kessler, K., Shapiro, K., Hommel, B., and Schnitzler, A. (2004). Modulation of long-range neural synchrony reflects temporal limitations of visual attention in humans. Proc. Natl. Acad. Sci. U.S.A. 101, 13050-13055.

Gruber, T., Muller, M. M., Keil, A., and Elbert, T. (1999). Selective visualspatial attention alters induced gamma band responses in the human EEG. Clin. Neurophysiol. 110, 2074-2085.

Haider, B., and McCormick, D. A. (2009) Rapid neocortical dynamics: cellular and network mechanisms. Neuron 62 , 171-189.

Hall, S. D., Holliday, I. E., Hillebrand, A., Singh, K. D., Furlong, P.L., Hadjipapas, A., and Barnes, G. R. (2005). The missing link: analogous human and primate cortical gamma oscillations. Neuroimage 26, 13-17.
Hasenstaub, A., Shu, Y., Haider, B., Kraushaar, U., Duque, A., and McCormick, D. A. (2005). Inhibitory postsynaptic potentials carry synchronized frequency information in active cortical networks. Neuron 47, 423-435.

Heeger, D. J., Simoncelli, E. P., and Movshon, J.A. (1996). Computational models of cortical visual processing. Proc. Natl. Acad. Sci. U.S.A. 93, 623-627.

Heekeren, H. R., Marrett, S., Bandettini, P. A., and Ungerleider, L. G. (2004). A general mechanism for perceptual decision-making in the human brain. Nature 431, 859-862.

Heekeren, H. R., Marrett, S., and Ungerleider, L. G. (2008). The neural systems that mediate human perceptual decision making. Nat. Rev. Neurosci. 9, 467-479.

Henrie, J. A., and Shapley, R. (2005). LFP power spectra in V1 cortex: the graded effect of stimulus contrast. $J$. Neurophysiol. 94, 479-490.

Hipp, J. F., Engel, A. K., and Siegel, M. (2011). Oscillatory synchronization in large-scale cortical networks predicts perception. Neuron 69, 387-396.

Hoogenboom, N., Schoffelen, J. M., Oostenveld, R., Parkes, L. M., and Fries, P. (2005).Localizing human visual gamma-band activity in frequency, time and space. Neuroimage. 29, 764-773.

Horwitz, G.D., Batista,A.P., and Newsome, W. T. (2004). Representation of an abstract perceptual decision in macaque superior colliculus. $J$. Neurophysiol. 91, 2281-2296.

Jensen, O., and Colgin, L. L. (2007). Cross-frequency coupling between neuronal oscillations. Trends Cogn. Sci. 11, 267-269.

Kable, J. W., and Glimcher, P. W. (2009). The neurobiology of decision: consensus and controversy. Neuron 63, 733-745.

Kaiser, J., Lennert, T., and Lutzenberger,W. (2007). Dynamics of oscillatory activity during auditory decision making. Cereb. Cortex 17, 2258-2267.

Kastner, S., and Ungerleider, L. G. (2000). Mechanisms of visual attention in the human cortex. Annu. Rev. Neurosci.23, 315-341.

Kayser, C., and König, P. (2004). Stimulus locking and feature selectivity prevail in complementary frequency ranges of V1 local field potentials. Eur. J. Neurosci. 19, 485-489.

Kim, J. N., and Shadlen, M. N. (1999). Neural correlates of a decision in the dorsolateral prefrontal cortex of the macaque. Nat. Neurosci. 2, 176-185.

König, P., Engel, A. K., and Singer, W. (1996). Integrator or coincidence detector? The role of the cortical neuron revisited. Trends Neurosci. 19, 130-137 
Kopell, N., Ermentrout, G. B., Whittington, M. A., and Traub, R. D. (2000). Gamma rhythms and beta rhythms have different synchronization properties. Proc. Natl. Acad. Sci. U.S.A. 97, 1867-1872.

Lakatos, P., Shah, A. S., Knuth, K. H., Ulbert, I., Karmos, G., and Schroeder, C. E. (2005). An oscillatory hierarchy controlling neuronal excitability and stimulus processing in the auditory cortex. J. Neurophysiol. 94, 1904-1911.

Lamme, V.A., and Roelfsema, P. R. (2000). The distinct modes of vision offered by feedforward and recurrent processing. Trends Neurosci. 23, 571-579.

Liu, J., and Newsome, W. T. (2006). Local field potential in cortical area MT: stimulus tuning and behavioral correlations. J. Neurosci. 26, 7779-7790.

Lo, C. C., and Wang, X. J. (2006). Corticobasal ganglia circuit mechanism for a decision threshold in reaction time tasks. Nat. Neurosci. 9, 956-963.

Machens, C. K., Romo, R., and Brody, C. D. (2005). Flexible control of mutual inhibition: a neural model of twointerval discrimination. Science 307, 1121-1124.

Mazurek, M. E., Roitman, J. D., Ditterich, J., and Shadlen, M. N. (2003). A role for neural integrators in perceptual decision making. Cereb. Cortex 13 , 1257-1269.

Miller, E. K., and Cohen, J. D. (2001). An integrative theory of prefrontal cortex function. Annu. Rev. Neurosci. 24, 167-202.

Montague, P. R., Hyman, S. E., and Cohen, J. D. (2004). Computational roles for dopamine in behavioural control. Nature 431, 760-767.

Moore, T., Armstrong, K. M., and Fallah, M. (2003). Visuomotor origins of covert spatial attention. Neuron 40 , 671-683.

Newsome, W. T., Britten, K. H., and Movshon, J. A. (1989). Neuronal correlates of a perceptual decision. Nature 341, 52-54.

Nienborg, H., and Cumming, B. G. (2009). Decision-related activity in sensory neurons reflects more than a neuron's causal effect. Nature 459, 89-92.

Palva, J. M., Palva, S., and Kaila, K. (2005). Phase synchrony among neuronal oscillations in the human cortex. $J$. Neurosci. 25, 3962-3972.

Pesaran, B., Nelson, M. J., and Andersen, R. A. (2008). Free choice activates a decision circuit between frontal and parietal cortex. Nature 453, 406-409.

Pesaran, B., Pezaris, J. S., Sahani, M., Mitra, P. P., and Andersen, R. A. (2002). Temporal structure in neuronal activity during working memory in macaque parietal cortex. Nat. Neurosci. 5, 805-811.
Pessoa, L., Gutierrez, E., Bandettini, P., and Ungerleider, L. (2002). Neural correlates of visual working memory: fMRI amplitude predicts task performance. Neuron 35, 975-987.

Pfurtscheller, G., Graimann, B., Huggins, J. E., Levine, S. P., and Schuh, L. A. (2003). Spatiotemporal patterns of beta desynchronization and gamma synchronization in corticographic data during self-paced movement. Clin. Neurophysiol. 114, 1226-1236.

Rees, G., Friston, K., and Koch, C. (2000). A direct quantitative relationship between the functional properties of human and macaque V5. Nat. Neurosci. 3, 716-723.

Ress, D., Backus, B. T., and Heeger, D. J. (2000). Activity in primary visual cortex predicts performance in a visual detection task. Nat. Neurosci. 3, 940-945.

Rickert, J., Oliveira, S. C., Vaadia, E., Aertsen, A., Rotter, S., and Mehring, C. (2005). Encoding of movement direction in different frequency ranges of motor cortical local field potentials. J. Neurosci. 25, 8815-8824.

Rodriguez, R., Kallenbach, U., Singer, W., and Munk, M. H. (2004). Short- and long-term effects of cholinergic modulation on gamma oscillations and response synchronization in the visual cortex. J. Neurosci. 24, 10369-10378.

Roitman, J. D., and Shadlen, M. N. (2002). Response of neurons in the lateral intraparietal area during a combined visual discrimination reaction time task. J. Neurosci. 22, 9475-9489.

Romo, R., and Salinas, E. (2003). Flutter discrimination: neural codes, perception, memory and decision making. Nat. Rev. Neurosci. 4, 203-218.

Saalmann, Y. B., Pigarev, I. N., and Vidyasagar, T. R. (2007). Neural mechanisms of visual attention: how top-down feedback highlights relevant locations. Science 316, 1612-1615.

Salinas, E., and Sejnowski, T. J. (2001). Correlated neuronal activity and the flow of neural information. Nat. Rev. Neurosci. 2, 539-550.

Scase, M. O., Braddick, O. J., and Raymond, J. E. (1996). What is noise for the motion system? Vision Res. 36, 2579-2586.

Schack, B., Vath, N., Petsche, H., Geissler, H. G., and Moller, E. (2002). Phasecoupling of theta-gamma EEG rhythms during short-term memory processing. Int. J. Psychophysiol. 44, 143-163.

Schall, J. D. (2001). Neural basis of deciding, choosing and acting. Nat. Rev. Neurosci. 2, 33-42.

Serences, J. T., and Boynton, G. M. (2007). The representation of behavioral choice for motion in human visual cortex. J. Neurosci. 27, 12893-12899.
Serences, J. T., and Yantis, S. (2006). Selective visual attention and perceptual coherence. Trends Cogn Sci. 10, 38-45.

Shadlen, M. N., Britten, K. H., Newsome, W. T., and Movshon, J. A. (1996). A computational analysis of the relationship between neuronal and behavioral responses to visual motion. J. Neurosci. $16,1486-1510$.

Shadlen, M. N., and Newsome, W. T. (2001). Neural basis of a perceptual decision in the parietal cortex (area LIP) of the rhesus monkey. J. Neurophysiol. 86, 1916-1936.

Shapley, R., Hawken, M., and Ringach, D. L. (2003). Dynamics of orientation selectivity in the primary visual cortex and the importance of cortical inhibition. Neuron 38, 689-699.

Shulman, G. L., Ollinger, J. M., Linenweber, M., Petersen, S. E., and Corbetta, M. (2001). Multiple neural correlates of detection in the human brain. Proc. Natl. Acad. Sci. U.S.A. 98 313-318.

Siegel, M., and Donner, T. H. (2010). "Linking band-limited cortical population activity to fMRI and behavior," in Integrating EEG and fMRI: Recording, Analysis, and Application, eds M. Ullsperger and S. Debener, (New York: Oxford University Press), 271-294.

Siegel, M., Donner, T. H., Oostenveld, R., Fries, P., and Engel, A. K. (2007). High-frequency activity in human visual cortex is modulated by visual motion strength. Cereb. Cortex 17, 732-741.

Siegel, M., Donner, T. H., Oostenveld, R., Fries, P., and Engel, A. K. (2008). Neuronal synchronization along the dorsal visual pathway reflects the focus of spatial attention. Neuron 60 709-719.

Siegel, M., and König, P. (2003). A functional gamma-band defined by stimulus-dependent synchronization in area 18 of awake behaving cats. J. Neurosci. 23, 4251-4260.

Siegel, M., Körding, K. P., and König, P. (2000). Integrating top-down and bottom-up sensory processing by somato-dendritic interactions. J. Comput. Neurosci. 8, 161-173.

Siegel, M., Warden, M. R., and Miller, E. K. (2009). Phase-dependent neuronal coding of objects in short-term memory. Proc. Natl. Acad. Sci. U.S.A. 106, 21341-21346.

Smith, P. L., and Ratcliff, R. (2004). Psychology and neurobiology of simple decisions. Trends Neurosci. 27, 161-168.

Steriade, M. (2000). Corticothalamic resonance, states of vigilance and mentation. Neuroscience 101, 243-276.

Sugrue, L. P., Corrado, G. S., and Newsome, W. T. (2005). Choosing the greater of two goods: neural currencies for valuation and decision making. Nat. Rev. Neurosci. 6, 363-375.

Tallon-Baudry, C., Bertrand, O., and Fischer, C. (2001). Oscillatory synchrony between human extrastriate areas during visual short-term memory maintenance. J. Neurosci. 21, RC177.

Taylor, K., Mandon, S., Freiwald, W. A., and Kreiter, A. K. (2005). Coherent oscillatory activity in monkey area $\mathrm{v} 4$ predicts successful allocation of attention. Cereb. Cortex 15, 1424-1437.

Tosoni, A., Galati, G., Romani, G. L., and Corbetta, M. (2008). Sensory-motor mechanisms in human parietal cortex underlie arbitrary visual decisions. Nat. Neurosci. 11, 1446-1453.

Usher, M., and McClelland, J. L. (2001). The time course of perceptual choice: the leaky, competing accumulator model. Psychol. Rev. 108, 550-592.

Van Der Werf, J., Jensen, O., Fries, P., and Medendorp, W. P. (2008). Gammaband activity in human posterior parietal cortex encodes the motor goal during delayed prosaccades and antisaccades. J. Neurosci. 28, 8397-8405.

Van Veen, B. D., van Drongelen, W., Yuchtman, M., and Suzuki, A. (1997). Localization of brain electrical activity via linearly constrained minimum variance spatial filtering. IEEE Trans. Biomed. Eng. 44, 867-880.

Varela, F., Lachaux, J.P., Rodriguez, E., and Martinerie, J. (2001). The brainweb: phase synchronization and largescale integration. Nat. Rev. Neurosci. 2, 229-239.

Vidal, J. R., Chaumon, M., O'Regan, J. K., and Tallon-Baudry, C. (2006). Visual grouping and the focusing of attention induce gamma-band oscillations at different frequencies in human magnetoencephalogram signals. J. Cogn Neurosci. 18, 1850-1862.

Wang, X. J. (2001). Synaptic reverberation underlying mnemonic persistent activity. Trends Neurosci. 24, 455-463.

Wang, X. J. (2002). Probabilistic decision making by slow reverberation in cortical circuits. Neuron 36, 955-968.

Wang, X. J. (2003). "Neural oscillations," in Encyclopedia of Cognitive Science, ed. L. Nadel (London: MacMillan), 272-280.

Wang, X. J. (2008). Decision making in recurrent neuronal circuits. Neuron 60, 215-234.

Womelsdorf, T., Schoffelen, J. M., Oostenveld, R., Singer, W., Desimone, R., Engel, A. K., and Fries, P. (2007). Modulation of neuronal interactions through neuronal synchronization. Science 316, 1609-1612.

Worden, M. S., Foxe, J. J., Wang, N., and Simpson, G. V. (2000). Anticipatory biasing of visuospatial attention 
indexed by retinotopically specific alpha-band electroencephalography increases over occipital cortex. J. Neurosci. 20, RC63.

Wyart, V., and Tallon-Baudry, C. (2008). Neural dissociation between visual awareness and spatial attention. $J$. Neurosci. 28, 2667-2679.

Wyart, V., and Tallon-Baudry, C. (2009). How ongoing fluctuations in human visual cortex predict perceptual awareness: baseline shift versus decision bias. J. Neurosci. 29, 8715-8725.

Yu,A. J., and Dayan, P. (2005). Uncertainty, neuromodulation, and attention. Neuron 46, 681-692.

Conflict of Interest Statement: The authors declare that the research was conducted in the absence of any commercial or financial relationships that could be construed as a potential conflict of interest.

Received: 02 June 2010; accepted: 17 February 2011; published online: 28 February 2011.

Citation: Siegel M, Engel AK and Donner TH (2011) Cortical network dynamics of perceptual decision-making in the human brain. Front. Hum. Neurosci. 5:21. doi: 10.3389/fnhum.2011.00021

Copyright (c) 2011 Siegel, Engel and Donner. This is an open-access article subject to an exclusive license agreement between the authors and Frontiers Media $S A$, which permits unrestricted use, distribution, and reproduction in any medium, provided the original authors and source are credited. 